PROCEEDINGS OF THE

AMERICAN MATHEMATICAL SOCIETY

Volume 125, Number 12, December 1997, Pages 3501-3508

S 0002-9939(97)04124-5

\title{
HNN-EXTENSIONS OF LIE ALGEBRAS
}

\author{
A. I. LICHTMAN AND M. SHIRVANI
}

(Communicated by Ken Goodearl)

\begin{abstract}
We define HNN-extensions of Lie algebras and study their properties. In particular, a sufficient condition for freeness of subalgebras is obtained. We also study differential HNN-extensions of associative rings. These constructions are used to give short proofs of Malcev's and Shirshov's theorems that an associative or Lie algebra of finite or countable dimension is embeddable into a two-generator algebra.
\end{abstract}

\section{INTRODUCTION}

The Higman-Neumann-Neumann extensions (HNN-extensions) of groups were first constructed in [9], and have been used since then for the proof of many embedding theorems, such as Higman, Neumann and Neumann's original theorem, that every countable group is embeddable into a group with two generators.

A remarkable analogy between the properties of amalgamated free products of groups and HNN-extensions has come to light since then, and a unified approach to the study of both constructions exists (see, e.g. Lyndon and Schupp [12], and Serre $[16])$.

The main goal of our paper is to construct HNN-extensions of Lie algebras and to study their properties. In fact, we consider the HNN construction for restricted Lie algebras, the case of ordinary Lie algebras being obtainable by obvious simplifications.

Let $L$ be a restricted $p$-algebra, $A$ a restricted subalgebra of $L$, and $\delta$ a derivation of $A$ into $L$. We define an HNN-extension $S=\langle L, A, t, \delta\rangle$ as the restricted Lie algebra generated by the algebra $L$ and an element $t$ subject to the relations $[t, a]=a \delta$ for all $a \in A$. (In fact we consider the more general case of a collection of subalgebras $A_{i} \quad(i \in I)$ and derivations $\delta_{i}: A_{i} \rightarrow L$ for every $i \in I$.) Our construction is completely similar to the group-theoretical one; as in the case of groups, we prove that $L$ is isomorphically embedded in $S$. Our main results on HNN-extensions of Lie algebras are Theorems 3 and 4 below.

Theorem 3. Let $S=\langle L, A, t, \delta\rangle$ be an HNN-extension of a restricted Lie algebra $L, \quad B$ a subalgebra of $S$ such that $B \cap A=0$. Let $B_{0}=B \cap L$. Then there exists a restricted free Lie algebra $C$ such that $B$ is isomorphic to a subalgebra of the free Lie sum $B_{0} * C$ (in the category of restricted Lie algebras).

Received by the editors March 22, 1996 and, in revised form, July 9, 1996.

1991 Mathematics Subject Classification. Primary 17B05; Secondary 16S10, 17 B01.

The first author was partially supported by the NSF Grant No. 144-F1181, and the second author by NSERC, Canada.

(C)1997 American Mathematical Society 
Theorem 3 implies the following result.

Theorem 4. Let $L$ be a restricted p-algebra, $A_{i}$ a family of subalgebras of $L$, and $\delta_{i}: A_{i} \rightarrow L$ derivations into $L$. Let $S=\left\langle L, A_{i}, t_{i}, \delta_{i}: i \in I\right\rangle$ be the corresponding HNN-extension. Let $B$ be a subalgebra of $S$ such that $B \cap L$ is free and $B \cap A=0$. Then $B$ is free.

Theorem 4 is the exact analogue of Kukin's Theorem on subalgebras of free Lie sums (see [11], or [3], Chapter 4). In the case of a single subalgebra $A \subseteq L$, the result follows from Theorem 3 and the Shirshov-Witt Theorem, that a subalgebra of a free Lie algebra is free.

In Section 4 we apply the HNN construction to give simple proofs of various known theorems. For example, we give a new proof of Shirshov's Theorem ([17], Theorem 4) that a Lie algebra of finite or countable dimension can be embedded into a 2-generator Lie algebra, and we give an easy proof of the theorem that every Lie algebra can be embedded into a simple Lie algebra.

The HNN construction for $L$ goes hand in hand with the corresponding construction for the $p$-envelope $U(L)$, so we begin by studying differential HNN-extensions of associative rings $R$, in a more general context than the one arising from the study of Lie algebras.

Let $R$ be an associative ring, $A_{i}(i \in I)$ a family of subrings of $R$, together with injective homomorphisms $\theta_{i}: A_{i} \rightarrow R$ and $\theta_{i}$-derivations $\quad \delta_{i}: A_{i} \rightarrow R$. We define the differential HNN-extension $\widetilde{R}=\left\langle R, A_{i}, t_{i}, \delta_{i}, \theta_{i}: i \in I\right\rangle$ by a universal mapping property, so $\widetilde{R}$ turns out to be generated by $R$ and additional elements $t_{i}$, subject to the relations $t_{i} a=\left(a \theta_{i}\right) t_{i}+a \delta_{i}$ for all $a \in A_{i}$ and all $i \in I$. If $R / A_{i}$ is a free left $A_{i}$-module for all $i$, then we show that $R$ is isomorphically embedded into $\widetilde{R}$. (The fact that a Lie algebra $L$ is embedded into $\langle L, A, t, \delta\rangle$ is an immediate consequence of this.) As another application, we give a simple proof of Malcev's Theorem (see [14]) that every associative algebra of finite or countable dimension is embeddable into a 2-generator algebra. One expects many of the properties of these HNN-extensions to be similar to the properties of the coproducts of rings ([2], and $[4]-[7])$.

Our differential HNN-extensions of associative rings should be compared with the HNN constructions of Macintyre [13] and Dicks [8]. The latter paper considers the case of associative rings $R$ and $K$, a pair of homomorphisms $\alpha$ and $\beta$ from $K$ to $R$, and a ring $\widetilde{R}$, generated by $R$ and elements $t$ and $t^{-1}$, subject to the relations $t^{-1} \alpha(k) t=\beta(k)$ for all $k \in K$.

\section{The associative CASE}

Let $R$ be an associative ring, $A_{i}(i \in I)$ a family of subrings of $R$, with injective homomorphisms $\theta_{i}: A_{i} \rightarrow R$ and, for each $i \in I$, a $\theta_{i}$-derivation $\quad \delta_{i}: A_{i} \rightarrow R$. An HNN-extension $\widetilde{R}=\left\langle R, A_{i}, t_{i}, \delta_{i}, \theta_{i}: i \in I\right\rangle$ is a ring $\widetilde{R}$ together with elements $t_{i}$ and a homomorphism $\psi: R \rightarrow \widetilde{R}$ such that

(1) $t_{i}(a \psi)-\left(a \theta_{i} \psi\right) t_{i}=a \delta_{i} \psi$ for all $a \in A_{i}$, all $i \in I$.

(2) Given any ring $S$, elements $\sigma_{i} \in S$, and a homomorphism $r \mapsto \bar{r}$ of $R$ into $S$ such that $\sigma_{i} \bar{a}-\left(\overline{a \theta_{i}}\right) \sigma_{i}=\overline{a \delta_{i}}$ for all $a \in A_{i}$ and all $i \in I$, there exists a unique homomorphism $\theta: \widetilde{R} \rightarrow S$ such that $t_{i} \theta=\sigma_{i}$ and $r \psi \theta=\bar{r}$ for all $r \in R$.

It is routine to see that $\widetilde{R}$ is generated by the $t_{i}$ and $R \psi$, and is unique. The existence of $\widetilde{R}$ may be proven by using generators and relations, by adding the 
relations

$$
t_{i} a-\left(a \theta_{i}\right) t_{i}=a \delta_{i} \quad \text { for all } a \in A_{i} \text {, all } i \in I,
$$

to a presentation of the coproduct of $R$ and the free algebra $R_{0}\left\langle t_{i}: i \in I\right\rangle$, where $R_{0}$ is the prime subring of $R$.

Assume that $R / A_{i}$ is a free left $A_{i}$-module for each $i$, and let $X_{i} \ni 1$ be a free basis of $R$ over $A_{i}$. A sequence is normal if it is of the form $t_{i_{1}} x_{1} t_{i_{2}} x_{2} \ldots t_{i_{r}} x_{r}$ (interpreted to be 1 if $r=0$ ), with $i_{\alpha} \in I$ and $x_{\alpha} \in X_{i_{\alpha}}$ for $1 \leq \alpha \leq r$. Let $V$ denote the set of all normal sequences.

Theorem 1. Let $R$ be an associative ring, $A_{i}$ a family of subrings of $R$, with injective homomorphisms $\theta_{i}: A_{i} \rightarrow R$ and, for each $i \in I$, a $\theta_{i}$-derivation $\delta_{i}$ : $A_{i} \rightarrow R$. Assume that $R / A_{i}$ is a free left $A_{i}$-module for all $i$, and let $(\widetilde{R}, \psi)$ be the corresponding HNN-extension as above. Then the map $\psi$ is an embedding of $R$ into $\widetilde{R}$, and identifying $R$ with $R \psi$, we have $\widetilde{R}=\oplus_{u \in V} R u$, where $V$ is the set of normal sequences. In particular, $\widetilde{R}$ is generated by $R$ and the $t_{i}$, subject to the relations $t_{i} a-\left(a \theta_{i}\right) t_{i}=a \delta_{i}$ for all $a \in A_{i}$ and all $i \in I$.

Proof. Let $Q=\oplus_{u \in V} R u$ be the free left $R$-module on $V$, so we have a faithful homomorphism $r \mapsto \bar{r}$ of $R$ into $S=\operatorname{End}_{\mathbb{Z}}(Q)$. Next, we define suitable $\sigma_{i} \in S$ for all $i \in I$. If $q \in Q$ is written as $q=\sum_{u \in V} \sum_{x \in X_{i}} a_{x, u} x u$ for (unique) $a_{x, u} \in A_{i}$, define

$$
\sigma_{i}(q)=\sum_{u \in V} \sum_{x \in X_{i}}\left(\left(a_{x, u} \theta_{i}\right) t_{i} x u+\left(a_{x, u} \delta_{i}\right) x u\right) .
$$

Observe that $\sum_{x \in X_{i}}\left(a_{x, u} \delta_{i}\right) x \in R$, while every $t_{i} x u \in V$ as $x \in X_{i}$. Clearly $\sigma_{i}$ is an additive map on $Q$. If $q$ is as above, then for any $a \in A_{i}(i \in I)$ we have

$$
\left(\sigma_{i} \bar{a}\right)(q)=\sigma_{i}\left(\sum_{u \in V} \sum_{x \in X_{i}} a a_{x, u} x u\right)=\sum_{u, x}\left[\left(\left(a a_{x, u}\right) \theta_{i}\right) t_{i} x u+\left(\left(a a_{x, u}\right) \delta_{i}\right) x u\right],
$$

while $\left(\overline{a \theta_{i}} \sigma_{i}\right)(q)=\sum_{u, x}\left(a \theta_{i}\right)\left[\left(a_{x, u} \delta_{i}\right) t_{i} x u+\left(a_{x, u} \delta_{i}\right) x u\right]$, and so $\left(\sigma_{i} \bar{a}-\overline{a \theta_{i}} \sigma_{i}\right)(q)=$ $\sum_{u, x}\left(a \delta_{i}\right) a_{x, u} x u=\overline{a \delta_{i}}(q)$. By property (2), there exists $\theta: \widetilde{R} \rightarrow S$ such that $t_{i} \theta=\sigma_{i}$ and $r \psi \theta=\bar{r}$ for all $r \in R$.

For $u=t_{i_{1}} x_{1} \ldots t_{i_{r}} x_{r} \in V$, write $u \psi=t_{i_{1}}\left(x_{1} \psi\right) \ldots t_{i_{r}}\left(x_{r} \psi\right) \in \widetilde{R}$. Then $u \psi \theta=$ $\sigma_{i} \bar{x}_{1} \ldots \sigma_{i_{r}} \bar{x}_{r}$, and induction on $r$ shows that $(u \psi \theta)(1)=u \in Q$. Plainly every element of $\widetilde{R}$ has the form $\sum_{u \in V}\left(r_{u} \psi\right)(u \psi)$. Then $\left(\sum_{u \in V}\left(\left(r_{u} \psi\right)(u \psi)\right) \theta(1)=\right.$ $\sum_{u}\left(\bar{r}_{u}(u \psi \theta)\right)(1)=\sum_{u} \bar{r}_{u}(u)=\sum_{u} r_{u} u \in Q$. Since $Q$ is free on $V$, the conclusions follow.

In the case of a single stable letter, $\widetilde{R}$ has the obvious $t$-filtration $\widetilde{R}_{-\infty}=\{0\} \subseteq$ $\widetilde{R}_{0} \subseteq \widetilde{R}_{1} \subseteq \ldots$, where $\widetilde{R}_{n}=\sum R u$, with the sum being over all $u=t x_{1} \ldots t x_{r}$ in $V$ with $r \leq n$. The following simple but useful result will not be required in the rest of the paper.

Lemma 1. If $R$ is a domain and $A$ is a division ring, then the $t$-filtration defines a valuation on $\left\langle R, A, t, \delta, i d_{A}\right\rangle$.

Proof. Clearly $\widetilde{R}_{0}=R$, and a standard argument shows that $\operatorname{gr}(\widetilde{R}) \cong R \sqcup_{A} A[t]$, the coproduct of $R$ and the polynomial ring $A[t]$. With the assumptions of the lemma, the above coproduct is a domain. The absence of zero-divisors in $\operatorname{gr}(\widetilde{R})$ is what defines a valuation. 


\section{HNN-EXTENSIONS OF LIE ALGEBRAS}

Let $L$ be a restricted Lie $p$-algebra, $A$ a subalgebra of $L$, with $U(L)$ and $U(A)$ the universal $p$-envelopes of $L$ and $A$ respectively (see [10], V, Theorem 12, or [15]). Let $\delta$ be a derivation of the $p$-algebra $A$ into $L$. We define the HNNextension $S=\langle L, A, t, \delta\rangle$ by the usual universal mapping property, as a retsricted $p$-Lie algebra $S$, together with a homomorphism $\psi$ of $L$ into $S$ and an element $t \in S$ such that $a \delta \psi=[t, a \psi]$ for all $a \in A$, and such that every homomorphism of $L$ into a restricted Lie algebra, in which $\delta$ becomes inner, factors uniquely through $S$. The existence is obvious (using generators and relations, for example). Now $\delta$ extends to a derivation of $U(A)$ into $U(L)$, and we may form the (associative) differential HNN-extension $\langle U(L), U(A), t, \delta\rangle$.

Theorem 2. Let $L$ be a restricted Lie p-algebra, A a p-subalgebra of $L, \delta$ a derivation of $A$ into $L$, and let $S=\langle L, A, t, \delta\rangle$. Then the universal p-envelope $U(S)$ is naturally isomorphic to the $H N N$-extension $\langle U(L), U(A), t, \delta\rangle$. In particular, $\psi$ embeds $L$ into $S$, and identifying $L$ with $L \psi, S$ is generated by $L$ and $t$, subject to the relations $[t, a]=a \delta$ for all $a \in A$.

Proof. Let $M=\langle U(L), U(A), t, \delta\rangle$, and let $R$ be an arbitrary associative algebra with a Lie homomorphism $S \longrightarrow R_{L}$ (the subscript denoting the Lie algebra of the ring). The restriction to $L$ extends to a homomorphism $U(L) \longrightarrow R$, which extends to a homomorphism $M \longrightarrow R$. This being compatible with the obvious map $S \longrightarrow M_{L}$, we have $U(S) \simeq M$ (cf. [10], V, Theorem 12), proving the first statement. As $U(L) / U(A)$ is a free left $U(A)$-module, Theorem 1 implies that $U(L)$ is embedded into $M$, and the rest of the result follows.

Clearly, the obvious analogue of Theorem 2 exists for the case of an arbitrary family of $p$-subalgebras $A_{i}$ and derivations $\delta_{i}: A_{i} \rightarrow L$.

Consider the situation of Theorem 2. The $t$-filtration of the HNN-extension $U(S)$ induces, in a natural way, a filtration in the restricted $p$-algebra $S$. It is easy to verify that the associated graded algebra $\operatorname{gr}(S)$ is again a restricted Lie $p$-algebra, which is naturally embedded into $\operatorname{gr}(U(S))$. The definition of the $t$-filtration implies that $\operatorname{gr}(L) \simeq L$, and the next result follows.

Lemma 2. With the notation of Theorem 2, the algebra $\operatorname{gr}(S)$ is isomorphic to the amalgamated Lie sum $L *_{A}(A \oplus K u)$ (in the category of restricted Lie p-algebras), where $u=t+L$ is the leading term of $t$ in $\operatorname{gr}(S)$.

From now on, we use left-normed commutators $\left[x_{1}, x_{2}, \ldots, x_{n}\right]$.

Lemma 3. Let $K$ be a field of characteristic $p>0, L$ a restricted Lie $K$-algebra, $A$ a subalgebra of $L$, and $\left\{e_{i}: i \in I\right\}$ a set of elements in $L$ which gives an ordered basis of the vector space $L / A$. Let $A_{1}=L \oplus K u$ be the direct sum of $A$ with a one-dimensional Lie algebra $K u$, and let $P=L *_{A} A_{1}$ be the free Lie sum of $L$ and $A_{1}$, with amalgamated subalgebra $A$. Let $U$ be the ideal of $P$ generated by the element $u$. Then the algebra $U$ is free on the generating set

$$
[u, \underbrace{e_{i_{1}}, \ldots, e_{i_{1}}}_{n_{1}} \underbrace{e_{i_{2}}, \ldots, e_{i_{2}}}_{n_{2}}, \ldots, \underbrace{e_{i_{k}}, \ldots, e_{i_{k}}}_{n_{k}}], i_{1}<\cdots<i_{k}, 0 \leq n_{j} \leq p-1, k \geq 0 .
$$

Further, the quotient algebra $P / U$ is isomorphic to $L$, so $P$ is a split extension $L \oplus U$ of $U$ by $L$. 
Proof. We consider first the free Lie sum $F=L * K u$. Take any basis $\left\{e_{j}: j \in J\right\}$ of $A$ over $K$, and adjoin it to the $\left\{e_{i}: i \in I\right\}$ to obtain a $K$-basis of $L$. It is well-known (see, e.g., [1], 2.6.5) that the set of elements

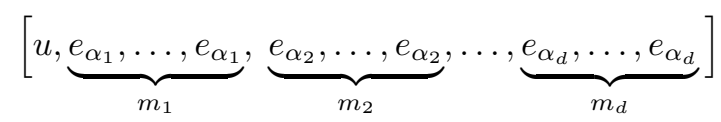

for $\alpha_{1}<\cdots<\alpha_{d}, \quad \alpha_{i} \in I, \quad 0 \leq m_{r} \leq p-1, \quad r=1, \ldots, d$, is a free system of generators of the Lie ideal $(u) \subseteq F$ (note that the order of the $\left\{e_{j}: j \in J\right\}$ has been extended arbitrarily to the system $\left\{e_{i}: i \in I\right\}$ ).

Now observe that $P$ is obtained from $F$ by adding all the relations $[u, a]=0$ for $a \in A$; hence, the kernel of the homomorphism $F \rightarrow P$ is the ideal $M$ of $F$ generated by all the elements $\left[u, e_{j}\right], \quad j \in J$. Therefore, $M$ contains all the elements from the system (2) which include some $e_{j} \quad(j \in J)$ in their representation, and so such elements form a free generating set for $M$. Thus the quotient algebra $U=(u) / M$ is freely generated by the remaining elements in (2), i.e., by the elements (1), and hence the first statement of the lemma is proven. The second statement follows from the isomorphisms $P / U \cong F /(u) \cong L$.

Proposition 1. Let $L$ be a Lie algebra, $S=\langle L, A, \delta, t\rangle$ an $\mathrm{HNN}$-extension of $L$. Assume that $\left\{e_{i}: i \in I\right\}$ is an ordered basis of the vector space $L / A$. Then the elements

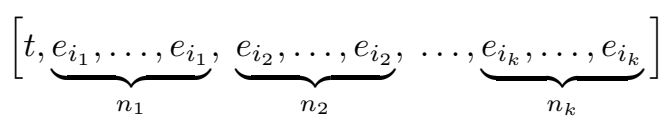

with $i_{1}<i_{2}<\cdots<i_{k}, \quad 0 \leq n_{j} \leq p-1, \quad j=1, \ldots, k$, freely generate a free subalgebra $T$ of $S$. Furthermore, $T \cap L=0$ and $S=L+T$.

Proof. Let $T$ be the subalgebra of $S$ generated by the elements (3). Lemma 2 implies that $\operatorname{gr}(S) \cong L *_{A} A_{1}$, where $A_{1}$ is a direct sum of $A$ and a one-dimensional algebra $K u$. Now the graded algebra $\operatorname{gr}(T)$, being the same as the ideal $U$ defined in Lemma 3 , is freely generated by the elements (1) of that lemma. A routine argument now shows that $T$ is freely generated by the elements (3). Next, $\operatorname{gr}(S)$ is generated by the subalgebras $\operatorname{gr}(L) \cong L$ and $\operatorname{gr}(T) \cong U$, and so $S$ is generated by $L$ and $T$. Finally, the $t$-degree of the elements in (3) is 1 , while the elements of $L$ have $t$-degree equal to zero, whence $L \cap T=0$.

Since the elements in (3) have $t$-degree equal to 1 , the $t$-grading on $T$ coincides with the natural grading defined by the free generators (3). Next, we define a grading on the algebra $P$ considered in Lemma 3 . To begin with, assign degree 1 to every element in the free generating set (1). This, of course, defines a grading and a degree function on the free algebra $U$. Assign degree zero to every element of $L$. Since every element of $P$ has a unique representation $x=y+v \quad(y \in L, v \in U)$, we obtain a grading on $P$. The next result follows from Lemmas 2 and 3 .

Lemma 4. Let $P$ be as in Lemma 3. Then the ideal $U$ and the subalgebra $L$ are homogeneous with respect to the above grading of $P$.

The following fact may be considered as an analogue of Proposition 4.7.1 in [3], which is one of the steps in the proof of Kukin's theorem on subalgebras of amalgamated Lie sums. 
Proposition 2. Let $B_{0}$ be a subalgebra of $L$ such that $B_{0} \cap A=0$. Then $\left[B_{0}, T\right] \subseteq$ $T$, and hence the subalgebra $\left\langle B_{0}, T\right\rangle$ generated by $B_{0}$ and $T$ is isomorphic to the split extension $B_{0} \oplus T$ of $T$ by $B_{0}$. Further, there exist free Lie subalgebras $T_{0}, T_{1}$ in $T$ such that $T \cong T_{0} * T_{1}$ and $B_{0} \oplus T \cong B_{0} * T_{0}$.

Proof. Take a vector space basis $X$ of $B_{0}$ and a subset $Y \subseteq L$ which gives a basis of $L /\left(A+B_{0}\right)$. Denote $X \cup Y$ by $\left\{e_{i}: i \in I\right\}$, ordered arbitrarily subject to $e_{i}<e_{j}$ if $e_{i} \in Y$ and $e_{j} \in X$. Let $T_{0}$ be the subalgebra of $T$, (freely) generated by those elements in (3) for which all $e_{i} \in Y$.

Consider the free Lie sum $B_{0} * T_{0}$ and its ideal $\left(T_{0}\right)$ generated by $T_{0}$. It is wellknown (see, for instance [1], 2.6.5) that $\left(T_{0}\right)$ is freely generated by all the elements (3), and that $B_{0} * T_{0}$ is a split extension of $\left(T_{0}\right)$ by $B_{0}$. Clearly the action of a basis element $e_{i} \in B_{0}$ on a free generator of $T_{0}$ in $B_{0} * T_{0}$ is the same as in the subalgebra $\left\langle B_{0}, T\right\rangle \subseteq S$. This proves that there exists an isomorphic mapping of $B_{0} * T_{0}$ onto $\left\langle B_{0}, T\right\rangle$, which maps $\left(T_{0}\right)$ onto $T$, and completes the proof.

Theorem 3. Let $S=\langle L, A, t, \delta\rangle$ be an $\mathrm{HNN}$-extension of a restricted Lie $p$-algebra $L$, and let $B$ be a subalgebra of $S$ such that $B \cap A=0$. Let $B_{0}=B \cap L$. Then there exists a free Lie p-algebra $C$ such that $B$ is isomorphic to a subalgebra of the free Lie sum $B_{0} * C$ (in the category of restricted Lie p-algebras).

Proof. By Proposition 2, it is sufficient to prove that $B \subseteq B_{0}+T$. Consider $\operatorname{gr}(S)$ and its subalgebra $\operatorname{gr}(B)$. Lemma 3 implies that $\operatorname{gr}(S)$ is a split extension of $U$ by $L$. From this, and Lemma 4 , it is easy to conclude that $\operatorname{gr}(B)$ is a subalgebra of the split extension of the ideal $\operatorname{gr}(B) \cap U$ by the subalgebra $B_{0}=\operatorname{gr}(B) \cap L$. Therefore,

$$
\operatorname{gr}(B) \subseteq B_{0}+U .
$$

It is now easy to show that $B \subseteq B_{0}+T$. Pick any $b \in B$ of degree $k \geq 1$. Then (4) implies the existence of $x \in T$ such that $b-x$ has degree $\leq k-1$. Since $B \supseteq B_{0}$, we obtain $b \in B_{0}+T$ by induction on $k$, as required.

Theorem 4. Let $L$ be a restricted p-algebra, $A_{i}$ a family of subalgebras, and $\delta_{i}$ : $A_{i} \rightarrow L$ derivations into $L$. Let $S=\left\langle L, A_{i}, t_{i}, \delta_{i}: i \in I\right\rangle$ be the corresponding HNN-extension. Let $B$ be a subalgebra of $S$ such that $B \cap A=0$ and $B \cap L$ is free. Then $B$ is free.

Proof. In the case of a single subalgebra, the result follows from Theorem 3 and the Shirshov-Witt Theorems ([3], Chapter 4) that a subalgebra of a free (restricted) Lie algebra is again free (restricted). The general case is easily obtained from the single variable case and Kukin's theorem ([11] or [3], Chapter 2), to the effect that if a subalgebra of an amalgamated Lie sum has zero intersection with the amalgamated subalgebra, and free intersection with the factors, then it is free.

\section{Applications}

We begin with a simple proof of Shirshov's Theorem [17], (see also [3], 2.18) that a Lie algebra of finite or countable dimension is embeddable into a 2-generator Lie algebra. In fact, the restricted case is just as easy to prove.

Theorem 5. Let $L$ be a restricted Lie p-algebra of finite or countable dimension over a field $K$. Then $L$ is embeddable into a two-generator restricted p-algebra over $K$. 
Proof. Let $\left\{e_{i}: i=1,2, \ldots\right\}$ be a basis of $L$ over $K$. Embed $L$ into the HNNextension $L_{1}=\left\langle L, t_{i}:\left[t_{i}, e_{1}\right]=e_{i}\right.$ for $\left.i=2,3, \ldots\right\rangle$. Then $L_{1}$ is generated by $e_{1}, t_{2}, t_{3}, \ldots$, and Theorem 4 (or an easy direct argument) implies that the subalgebra $T=\left\langle t_{2}, t_{3}, \ldots\right\rangle$ is freely generated by $t_{2}, t_{3}, \ldots$. Define a derivation $\delta: T \rightarrow L_{1}$ as follows: $t_{i} \delta=t_{i+1}$ for all $i$ if $\operatorname{dim} L$ is infinite, while if $\operatorname{dim} L=n$, then $t_{i} \delta=t_{i+1}$ for $i \leq n-1$, and $t_{n} \delta=0$. Then $L_{1}$ embeds into the HNN-extension $L_{2}=\left\langle L_{1}, T, u, \delta\right\rangle$, and clearly $L_{2}$ is generated by $e_{1}, t_{2}$, and $u$. The subalgebra $A=\left\langle u, t_{2}\right\rangle$ is free on $u$ and $t_{2}$. If $\delta^{\prime}: A \rightarrow L_{2}$ is the derivation $u \delta^{\prime}=t_{2}, \quad t_{2} \delta^{\prime}=e_{1}$, then $L_{2}$ embeds into the HNN-extension $\left\langle L_{2}, A, v, \delta^{\prime}\right\rangle$, which is generated by $u$ and $v$, as required.

Theorem 6 (see [3], Chapter 3). Let L be a restricted Lie p-algebra over a field $K$. Then $L$ is embeddable into a simple restricted Lie p-algebra $H$. Further, if $\operatorname{dim}_{K}(L)$ is at most countably infinite, then $\operatorname{dim}_{K}(H)$ is countably infinite.

Proof. Let $\left\{\left(u_{i}, v_{i}\right): i \in I\right\}$ be the set of all ordered pairs of elements of $L$. Embed $L$ into the HNN-extension $H_{1}=\left\langle L, t_{i}:\left[t_{i}, u_{i}\right]=v_{i}\right.$ for all $\left.i \in I\right\rangle$. The process may be repeated to yield a chain $L \subseteq H_{1} \subseteq H_{2} \subseteq \ldots$. It is easy to see that $H=\cup_{j=1}^{\infty} H_{j}$ satisfies all the conclusions of the theorem.

Our final application is to associative rings.

Theorem 7 (Malcev [14], or see [3], Chapter 2). Let $R$ be an associative algebra which has finite or countable dimension over a field $K$. Then $R$ is embeddable into a two-generator $K$-algebra.

Proof. Let $\left\{e_{i}: i=1,2, \ldots\right\}$ be a basis of $R$ over $K$, and let $S=R \sqcup_{K} K[t]$ be the coproduct of $R$ with the polynomial ring $K[t]$. If $u_{i}=e_{i}+t$, then it is evident that $S=R \sqcup_{K} K\left[u_{i}\right]$ for every $i$, and $K\left[u_{i}\right]$ is the polynomial algebra on $u_{i}$. In particular, it is clear that $S$ is a free left module over $K[t]$ or the $K\left[u_{i}\right]$ (use the normal form theorem for coproducts, [2], or [5]-[7]). Since the elements $u_{i}$ and $t$ generate $S$, the same argument as in Theorem 5 yields that $S$ is embeddable into a two-generator algebra.

\section{ACKNOWLEDGMENT}

We would like to thank the referee for a very careful reading of the paper, and many useful and perceptive comments, which greatly improved the exposition.

\section{REFERENCES}

1. Yu. A. Bakhturin, Identical Relations in Lie Algebras, VNU Scientific Press, Utrecht, 1987. MR 88f: 17032

2. G.M. Bergman, Modules over coproducts of rings, Trans Amer. Math.Soc. 200 (1974), 1-32. MR 50:9970

3. L.A. Bokut and G.P. Kukin, Algorithmic and Combinatorial Algebra, Kluwer, 1994. MR 95i: 17002

4. P.M. Cohn, Skew fields: theory of general division rings, Cambridge University Press, New York, 1995. MR 97d:12003

5. P.M. Cohn, On the free product of associative rings Math. Z. 71 (1959), 380-398. MR 21:5648

6. P.M. Cohn, On the free product of associative rings, II, Math. Z. 73 (1960), 433-456. MR 22: 4747

7. P.M. Cohn, On the free product of associative rings, III, J. Algebra 8 (1968), 376-383. MR 36:5170

8. W. Dicks, The HNN construction for rings, J. Algebra 81 (1983), 434-487. MR 85c:16005 
9. G. Higman, B.H. Neumann and H. Neumann, Embedding theorems for groups, J. London Math. Soc. 24 (1949), 247-254. MR 11:322d

10. N. Jacobson, Lie Algebras, Dover, New York, 1979. MR 80k:17001

11. G.P. Kukin, Subalgebras of a free Lie sum with an amalgamated subalgebra, Algebra i Logika 11 (1972), 59-86 (In Russian). MR 46:9133

12. R.C. Lyndon and P.E. Schupp, Combinatorial Group Theory, Springer, Berlin, 1977. MR 58:28182

13. A. Macintyre, Combinatorial problems for skew fields, I. Analogue of Britton's lemma, and results of Adjan-Rabin type, Proc. London Math. Soc. (3) 39 (1979), 211-236. MR 81h:03092

14. A.I. Malcev, On representations of infinite algebras, Mat. Sb. 13 (1943), 263-285 (In Russian). MR 6:116c

15. G.B. Seligman, Modular Lie Algebras, Springer, Berlin, 1967. MR 39:6933

16. J.-P. Serre, Trees, Springer, Berlin, 1980. MR 82c:20083

17. A.I. Shirshov, On free Lie rings, Mat. Sb. 45 (1958), 13-21.

Department of Mathematics, University of Wisconsin-Parkside, Kenosha, Wisconsin $53141-2000$

E-mail address: lichtman@cs.uwp.edu

Department of Mathematical Sciences, University of Alberta, Edmonton, Alberta, CANADA T6G 2G1

E-mail address: mazi@schur.math.ualberta.ca 